\title{
BMJ Open Delirium and neuropsychological outcomes in critically Ill patients with COVID-19: a cohort study
}

\author{
Jacqueline Ragheb, ${ }^{1}$ Amy McKinney, ${ }^{1}$ Mackenzie Zierau, ${ }^{1}$ Joseph Brooks, ${ }^{1}$ \\ Maria Hill-Caruthers, ${ }^{1}$ Mina Iskander, ${ }^{2}$ Yusuf Ahmed, ${ }^{1}$ Remy Lobo, ${ }^{3}$ \\ Graciela Mentz, ${ }^{1}$ Phillip E Vlisides (D) ${ }^{1,4}$
}

To cite: Ragheb J, McKinney A,

Zierau M, et al. Delirium and neuropsychological outcomes in critically III patients with COVID-19: a cohort study. BMJ Open 2021;11:e050045. doi:10.1136/ bmjopen-2021-050045

- Prepublication history and additional supplemental material for this paper are available online. To view these files, please visit the journal online (http://dx.doi.org/10.1136/ bmjopen-2021-050045)

JR and AM contributed equally.

Received 09 February 2021 Accepted 05 September 2021

A) Check for updates

C Author(s) (or their employer(s)) 2021. Re-use permitted under CC BY-NC. No commercial re-use. See rights and permissions. Published by BMJ.

${ }^{1}$ Anesthesiology, Michigan Medicine, Ann Arbor, Michigan USA

${ }^{2}$ New York Medical College, Valhalla, New York, USA ${ }^{3}$ Radiology, Michigan Medicine, Ann Arbor, Michigan, USA

${ }^{4}$ Center for Consciousness

Science, University of Michigan

Medical School, Ann Arbor,

Michigan, USA

Correspondence to

Phillip E Vlisides;

pvliside@med.umich.edu

\section{ABSTRACT}

Objective To characterise the clinical course of delirium for patients with COVID-19 in the intensive care unit, including postdischarge neuropsychological outcomes. Design Retrospective chart review and prospective survey study.

Setting Intensive care units, large academic tertiary-care centre (USA).

Participants Patients ( $n=148$ ) with COVID-19 admitted to an intensive care unit at Michigan Medicine between 1 March 2020 and 31 May 2020 were eligible for inclusion. Primary and secondary outcome measures Delirium was the primary outcome, assessed via validated chart review method. Secondary outcomes included measures related to delirium, such as delirium duration, antipsychotic use, length of hospital and intensive care unit stay, inflammatory markers and final disposition. Neuroimaging data were also collected. Finally, a telephone survey was conducted between 1 and 2 months after discharge to determine neuropsychological function via the following tests: Family Confusion Assessment Method, Short Blessed Test, Patient-Reported Outcomes Measurement Information System Cognitive Abilities 4a and Patient-Health Questionnaire-9.

Results Delirium was identified in 108/148 (73\%) patients, with median (IQR) duration lasting 10 (4-17) days. In the delirium cohort, $50 \%$ (54/108) of patients were African American and delirious patients were more likely to be female $(76 / 108,70 \%)$ (absolute standardised differences $>0.30$ ). Sedation regimens, inflammation, delirium prevention protocol deviations and hypoxicischaemic injury were likely contributing factors, and the most common disposition for delirious patients was a skilled care facility $(41 / 108,38 \%)$. Among patients who were delirious during hospitalisation, 4/17 (24\%) later screened positive for delirium at home based on caretaker assessment, 5/22 (23\%) demonstrated signs of questionable cognitive impairment or cognitive impairment consistent with dementia and 3/25 (12\%) screened positive for depression within 2 months after discharge.

Conclusion Patients with COVID-19 commonly experience a prolonged course of delirium in the intensive care unit, likely with multiple contributing factors. Furthermore, neuropsychological impairment may persist after discharge.
Strengths and limitations of this study

- The validated chart review method increases confidence in the delirium findings reported.

- Granular details included (ie, inflammatory profiles, neuroimaging findings and postdischarge neuropsychological function) provide a comprehensive assessment of delirium phenotype in this patient population along with related complications.

- As a single-centre study, findings are restricted to the institution included.

- Many patients were lost to follow-up after hospital discharge.

\section{INTRODUCTION}

The outbreak of SARS-CoV-2, the virus that causes COVID-19, emerged as a public health threat in December 2019 and was declared a pandemic by WHO in March 2020. Major neurological complications, such as encephalopathy, strokes, seizures and ataxia, have all been observed. ${ }^{1-5}$ Delirium appears to be a common complication, with previous investigations demonstrating an incidence of approximately $65 \%-80 \%$ in the intensive care unit (ICU). ${ }^{14}$ Delirium may occur due to direct coronavirus invasion of the central nervous system, ${ }^{6}$ and systemic inflammatory responses may further exacerbate neurocognitive impairment. In the ICU, multiple delirium risk factors are often present and can increase risk in an additive manner. ${ }^{7}$ Delirium is also associated with prolonged hospitalisation, long-term cognitive and functional impairment and increased mortality. ${ }^{8-10}$ As such, there is a critical need to improve understanding of this syndrome in patients with COVID-19.

While a high incidence of delirium has been reported in COVID-19 patients, fundamental questions persist. The clinical course of delirium, including average duration and postdischarge cognitive trajectory, remains 
incompletely understood. Pathophysiologic drivers of delirium require advanced understanding, and the extent to which standard prevention protocols are implemented is unclear. Such detailed understanding will contribute to delirium phenotyping of COVID-19 patients and provide insight into the clinical and neurocognitive burden associated with COVID-19. In this context, the objective of this study was to determine granular details associated with delirium in ICU patients with COVID-19. Specifically, the clinical course of delirium, presence of exacerbating factors, nature of prevention strategy implementation and postdischarge cognitive outcomes were all characterised.

\section{METHODS}

\section{Study design and overview}

This was a single-centre cohort study from Michigan Medicine. Detailed chart review data were collected from critically ill patients with COVID-19 (1 March 2020 to 31 May 2020), and postdischarge telephone surveys were conducted to test neuropsychological function after discharge. All study operations were conducted at Michigan Medicine, Ann Arbour MI USA, and approval was obtained from the University of Michigan Medical School Institutional Review Board (HUM00182646). A Health Insurance Portability and Accountability Act waiver was granted to retrospectively review patient medical records, and informed consent was not required for retrospective chart review. Patients who agreed to complete telephone surveys after discharge were consented over the telephone prior to survey administration using a comprehensive consent document. A waiver of documentation of consent was approved in conjunction with Institutional Review Board approval and as required by US Department of Health and Human Services regulations and policy. Finally, the Strengthening the Reporting of Observational Studies in Epidemiology checklist is included in the supplemental online material (online supplemental table 1). These guidelines provide reporting standards for observational studies. ${ }^{11}$

\section{Eligibility criteria}

All patients with a COVID-19 diagnosis admitted to a Michigan Medicine ICU between 1 March 2020 and 31 May 2020 were eligible for study inclusion. ICU patients admitted during this time, without a diagnosis of COVID19 , were not eligible for study inclusion.

\section{Outcomes}

The primary outcome was delirium presence (yes/no, $\%)$ at any point during admission (see 'Data collection' section for delirium assessment details). Several secondary outcomes were also collected in relation to delirium and overall clinical trajectory. These outcomes included the following: duration of delirium (days), antipsychotic administration (which may serve as a surrogate marker for hyperactive delirium and/or agitation), length of hospital stay, length of ICU stay, number of days requiring ventilator support, haemodialysis (given the association between renal injury an delirium), ${ }^{12}$ inflammatory laboratory values that have been implicated with COVID-19 and cognitive dysfunction (white blood cell count, c-reactive protein, ferritin, lactate dehydrogenase, d-dimer and interleukin-6), ${ }^{245}$ new psychiatry consults, new antidepressant use (given the possibility of major depressive disorder with critical illness) ${ }^{13}$ and final disposition (eg, home, long-term care facility and death). Delirium prevention strategies, based on the ABCDEF ICU liberation bundle, ${ }^{14}{ }^{15}$ were also recorded. These included the following: structured mobility exercises, placing familiar objects from home at the bedside, promoting use of visual and hearing aids and spontaneous awakening/breathing trials. The total number of times a prevention strategy was charted was recorded for each patient, and this number was divided by the expected number of times that intervention should have occurred based on length of ICU stay and protocolised schedule. This provided the estimated compliance rate for each intervention. Neuroimaging data were also collected and reviewed. There was no neuroimaging protocol in place for patients with COVID19. Rather, neuroimaging was ordered at the discretion of clinical care teams as indicated.

Finally, a telephone survey was conducted after hospital discharge to determine whether subjective or objective signs of cognitive impairment were present. The initial phone call was placed between 30 and 60 days postdischarge. Twenty patients did not return phone calls until after 60 days, and the average length of time between discharge and survey administration was 83 days. Telephone surveys were conducted by a member of the research team (AM) with formal training in the Confusion Assessment Method for delirium. ${ }^{16}$ Phone calls were placed between the hours of 9:00 AM and 4:00 PM, Monday through Saturday and a total of three telephone contact attempts were made before considering loss to follow-up. Voicemail messages were left after each phone call. During telephone interviews, the following tests were conducted: the Patient-Reported Outcomes Measurement Information System (PROMIS) ${ }^{17}$ Cognitive Function Abilities 4a, Short Blessed Test (score 0-4=normal cognition, score 5-9=questionable impairment, score $\geq 10$ $=$ impairment consistent with dementia),$^{18}$ Family Confusion Assessment Method (FAM-CAM) for delirium ${ }^{19}$ and the Patient Health Questionnaire-9 (scores $\geq 10$ were considered positive screens for depression).$^{2021}$

\section{Data collection}

Screening for eligible patients was first performed via DataDirect, a software tool from the University of Michigan Office of Research that enables research teams to retrospectively search for patient cohorts. Charts that screened positive were then manually reviewed by study team members to confirm study eligibility.

Charts were then reviewed in further detail for outcome abstraction. Delirium was defined by the presence of either 
of the following criteria: (1) a positive CAM screen, ${ }^{22}$ as conducted by the bedside nurse, or (2) the presence of an acute confusional state, as documented in the medical record and elucidated via validated, standardised, chart review method. ${ }^{23}$ In terms of CAM screening, this is conducted every 12 hours by the bedside ICU nurse per hospital protocol. Nurses at our institution receive formal training in the CAM for delirium during their clinical training. For the chart review method, members of the research team reviewed patient charts with the aim of identifying any instance of an acute confusional state, which would count as an episode of delirium. The methodology for defining an acute confusion state is also drawn from the CAM, ${ }^{22}$ which assesses for acute changes in cognition, fluctuating course, inattention, altered levels of consciousness and disorganised thinking. This was the core set of delirium symptoms in this cohort, and hyperactive states (eg, agitation) were reported as well. If these symptoms were present for a given patient, the patient was considered as having delirium, and the source of information was recorded along with the date and time. The total number of days with acute confusion was also included in the instrument, along with any evidence of reversibility or improvement of the confusion state. Thus, overall, a case of delirium was counted either for (1) a positive CAM screen or (2) an acute confusional state documented in the medical record as abstracted by the chart review method described. Other clinical outcomes, along with laboratory values, were collected directly from the charts. Neuroimaging studies were manually reviewed by a board-certified radiologist with a Certificate of Added Qualification in neuroradiology (RL).

\section{Patient and public involvement}

Neither patients nor the public were involved in this research.

\section{Statistical analysis}

Analyses were performed using IBM SPSS V.27 (Armonk, NY USA) and SAS V.9.4 (SAS Institute, Cary, NC USA). Exploratory data analysis techniques were used to assess the distribution of dependent measures for determining the appropriate analytical strategy. The Shapiro-Wilk test was used to assess the distribution of continuous outcomes, and Independent t-tests or Mann-Whitney U tests were used as appropriate. Mean (SD) or median (IQR) was reported for parametric and non-parametric data, respectively. For binary outcomes and proportions, The $\mathrm{X}^{2}$ Test or Fisher's Exact Test were used, as appropriate. Absolute standardised differences were calculated for determining differences in baseline characteristics between groups, with differences $>0.20$ considered to be imbalanced. The threshold for significance was set to $p<0.05$ across all tests otherwise. For postdischarge cognitive outcomes, descriptive statistics were reported with no missing data plan for loss to follow-up. As a descriptive study, with chart data available for all patients, there was no missing data analysis.

\section{RESULTS}

In total, 148 patients were included in the final cohort analysis (online supplemental figure 1). Baseline characteristics are presented in table 1 . The majority of patients were African American and non-Hispanic, and the most common comorbidities were hypertension, diabetes mellitus and obesity. Absolute standardised differences between delirium and non-delirium groups were largest $(>0.30)$ for sex, race and weight. The highest proportion of patients in the delirium group was African American $(\mathrm{n}=54,50 \%)$.

\section{Delirium and neuropsychological outcomes}

Delirium incidence was high in the cohort $(108 / 148$, $73 \%$ ), and median (IQR) duration was 10 (4-17) days (table 2). Delirium prevention activities occurred relatively infrequently, with estimated unit protocol compliance rates less than $50 \%$ for each intervention reported (see table 2 legend for description of protocol activity schedule). The mobility exercise activity compliance rate $(\%)$ was significantly lower in the delirium group $(37 \%(26-55))$ compared with the non-delirium group (62\% (31-152); $\mathrm{p}=0.009)$. Likewise, daily promotion of visual and hearing aids occurred less frequently in the delirium group (27\% (13-63)) compared with the nondelirium group (77\% (14-213); $\mathrm{p}=0.005)$. New antidepressant use was more common for those with delirium $(27 / 108,25 \%)$ compared with those without delirium $(3 / 40,7.5 \%$; $p=0.01)$. Similarly, a psychiatry consult was obtained for 21/108 (19\%) delirious patients compared with $0 / 40(0 \%)$ in the non-delirium group $(\mathrm{p}=0.003)$. Finally, no evidence of delirium reversal or improvement was reported for more than $30 \%$ of patients during index hospitalisation.

\section{Hospitalisation and postdischarge outcomes}

Median length of hospitalisation was 25 (13-48) days, and median length of ICU stay was 15 (7-31) days across the cohort (table 3). Length of hospitalisation, ICU length of stay and duration of mechanical ventilation were all significantly prolonged in patients experiencing delirium (table 3). Correspondingly, sedative-hypnotic use was higher in patients with delirium. Delirious patients demonstrated higher white blood cell counts, c-reactive protein levels and d-dimer levels compared with non-delirious patients. Less than half of patients were ultimately discharged home, and the most common disposition for those with delirium was a skilled care facility $(41 / 108,38 \%)$ after discharge (table 3$)$.

Neuropsychological outcomes after discharge are reported in table 4 . Among patients who were still alive and available to complete survey materials, nearly $25 \%$ of patients $(4 / 17)$ scored positive for delirium based on family assessment (FAM-CAM), and all of these patients were delirious during hospitalisation. Similarly, approximately $23 \%$ of patients (5/22) demonstrated either questionable impairment or impairment consistent with dementia based on the Short Blessed Test, and all five of 
Table 1 Baseline characteristics

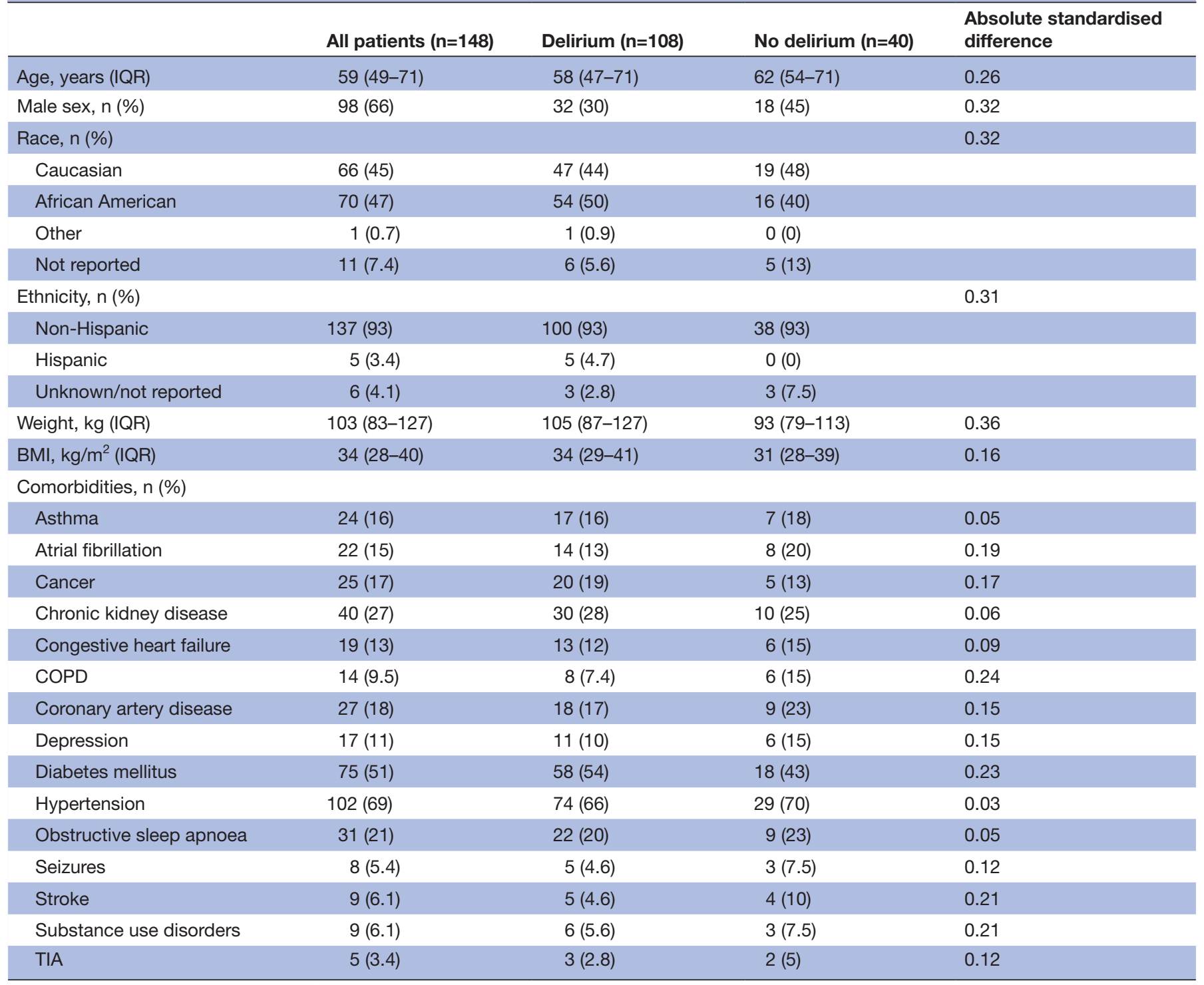

Median (IQR) data presented.

BMI, body mass index; COPD, chronic obstructive pulmonary disease; TIA, transient ischaemic attack.

these patients were also delirious during hospitalisation. Of note, three of these five patients also screened positive for delirium based on the FAM-CAM. Finally, 12\% of patients $(3 / 25)$ screened positive for depression after discharge. The three patients who screened positive also experienced delirium during ICU admission.

\section{Neuroradiological findings}

In total, 47 patients underwent neuroimaging during hospitalisation. Neuroimaging studies were ordered at the discretion of clinical care teams based on clinical assessment. No standardised neuroimaging protocols were in place for patients with COVID-19. The majority of imaging results were unremarkable or demonstrated incidental findings unrelated to COVID-19. However, some notable findings were present. A brain MRI was ordered for a patient with COVID-19 pneumonia and worsening encephalopathy (ie, no response to commands or noxious stimulus). Imaging revealed abnormal fluid attenuated inversion recovery hyperintensity affecting the occipital and temporal lobes (figure 1A,B), microhaemorrhage in the splenium of the corpus callosum (figure 1B,C) and posterior leptomeningeal enhancement figure $1 \mathrm{C}, \mathrm{D})$, suggestive of encephalitis. A brain MRI was ordered for another patient presenting with seizures and recent COVID-19 diagnosis. Results revealed diffuse dural thickening and enhancement (online supplemental figure 2A) 1 day prior to positive COVID-19 testing. The differential diagnosis included intracranial hypotension, inflammation, infection and neoplastic processes. No definitive diagnosis was reached, though this enhancement resolved approximately 1 month later (online supplemental figure 2B). Finally, one patient demonstrated 
Table 2 Delirium and neuropsychological outcomes

\begin{tabular}{|c|c|c|c|c|}
\hline & All patients $(n=148)$ & $\begin{array}{l}\text { Delirium } \\
(n=108)\end{array}$ & $\begin{array}{l}\text { No delirium } \\
(n=40)\end{array}$ & $P$ value \\
\hline \multicolumn{5}{|l|}{ Delirium measures } \\
\hline Duration of delirium, days (IQR) & $10(4-17)$ & $10(4-17)$ & - & - \\
\hline Agitation $\mathrm{n},(\%)$ & - & $54(50)$ & - & - \\
\hline \multicolumn{5}{|c|}{ ICU liberation bundle activity compliance rate, \% (IQR) } \\
\hline Mobility exercises & $40(28-67)$ & $37(26-55)$ & $62(31-152)$ & 0.009 \\
\hline Familiar objects at bedside & $14(4.4-31)$ & $14(5.9-25)$ & $18(0-62)$ & 0.38 \\
\hline Daily visual and hearing aids & $33(13-76)$ & $27(13-63)$ & $77(14-213)$ & 0.005 \\
\hline New psychiatry consults, n (\%) & $21(14)$ & $21(19)$ & $0(0)$ & 0.003 \\
\hline
\end{tabular}

Delirium prevention measures are based on the standard ICU liberation bundle protocols (see text for details). Per institutional protocol, clinicians conduct mobility exercises three times daily, place familiar objects at the bedside once daily, promote visual and hearing aid use daily and conduct daily spontaneous awakening/breathing trials daily (if eligible). Given this schedule, compliance/occurrence rates (\%) were calculated for each patient by calculating the total number of activities charted divided by the total number expected based on length of ICU stay (day of ICU discharge was not counted). ICU, intensive care unit.

diffuse parenchymal abnormalities on MRI suggestive of bilateral hypoxic-ischaemic injury after multiple cardiopulmonary arrests (online supplemental figure 3). A non-contrast head CT 2 weeks later demonstrated poor sulcation bilaterally, suggesting global hypoxic-ischaemic injury (online supplemental figure 4).

\section{DISCUSSION}

In a cohort of ICU patients with COVID-19, delirium was a common complication, affecting more than $70 \%$ of patients. Furthermore, delirium was associated with prolonged hospitalisation, increased length of ICU stay, discharge to skilled care facilities and positive screens for neuropsychological impairment during the months after discharge. Delirium occurred in the setting of multiple sedative-hypnotic agents, acute inflammatory responses, deviation from delirium prevention protocols and cerebrovascular events, which are all factors that could have further catalysed delirium precipitation. ICU liberation activities were infrequently implemented compared with the protocolised frequency expected. Overall, the burden of cognitive impairment was high in patients with COVID19 , as was the risk of related complications.

These results align with previous data demonstrating a high incidence of delirium in critically ill patients with COVID-19. ${ }^{1-4}$ Moreover, the median duration of delirium (10 days) is relatively long compared with other critically ill populations. ${ }^{24-27}$ Our findings also highlight the multifactorial nature of delirium risk factors. In terms of demographics, $50 \%$ of patients in the delirium group were African American. The proportion of African American patients admitted to the ICU and experiencing delirium was disproportionately high compared with our expected hospital demographic profile. COVID-19 has adversely, and disproportionately, impacted racial and ethnic minority communities, ${ }^{28} 29$ and our results further suggest an increased risk of attendant complications (eg, delirium) during hospitalisation. Efforts to reduce racial healthcare disparities may thus, by extension, mitigate risk of delirium and related consequences of COVID-19. Patients experiencing delirium also demonstrated significantly increased weight, and obesity may drive organ dysfunction via immune system dysregulation. ${ }^{30}$ Additionally, there was a disproportionate number of female patients in the delirium group (absolute standardised difference $>0.30$ ). These results are discrepant from a prior case series of critically ill patients with COVID-19 demonstrating an increased risk of delirium with male patients. ${ }^{4}$ Male sex has also been identified as an independent risk factor for delirium in other patient populations, possibly due to underlying comorbidity severity. ${ }^{31} 32$ Whether the findings in this study are spurious or reflect an underlying biological phenomenon is unclear. Further investigation is warranted to improve understanding of the impact that such demographic factors on delirium risk in patients with COVID-19.

Cognitive dysfunction may also occur as a result of direct coronavirus invasion of the central nervous system ${ }^{6}$ 
Table 3 Hospitalisation

\begin{tabular}{|c|c|c|c|c|}
\hline & All patients $(n=148)$ & Delirium $(n=108)$ & No delirium $(n=40)$ & $P$ value \\
\hline Length of hospitalisation, days (IQR) & $25(13-48)$ & $31(18-52)$ & $11(7-25)$ & $<0.001$ \\
\hline Length of ICU stay, days (IQR) & $15(7-31)$ & $19(12-38)$ & $4(2-8)$ & $<0.001$ \\
\hline Ventilator time, days, (IQR) & $12(3-28)$ & $18(10-29)$ & $0(0-7)$ & $<0.001$ \\
\hline Required haemodialysis, n (\%) & $45(30)$ & $40(37)$ & $5(13)$ & 0.004 \\
\hline \multicolumn{5}{|l|}{ Sedative-hypnotics, n (\%) } \\
\hline Propofol & $113(76)$ & $98(91)$ & $15(38)$ & $<0.001$ \\
\hline Midazolam & $87(59)$ & 75 (69) & $12(30)$ & $<0.001$ \\
\hline Dexmedetomidine & $97(66)$ & $89(82)$ & $8(20)$ & $<0.001$ \\
\hline Lorazepam & $58(39)$ & $52(49)$ & $6(15)$ & $<0.001$ \\
\hline \multicolumn{5}{|l|}{ Laboratories } \\
\hline WBC $(n=148) 4.0-10.0(K / \mu L)$ & $10.1(7.8-13.9)$ & $11.3(8.4-15.1)$ & $8.7(6.3-10.9)$ & 0.002 \\
\hline C-reactive protein $(\mathrm{n}=145)$ 1.0-3.0 (mg/L) & $10.2(5.1-18.0)$ & $11.7(5.3-20.7)$ & $8.9(4.3-13.9)$ & 0.03 \\
\hline Ferritin $(\mathrm{n}=147) 18.0-320.0(\mathrm{ng} / \mathrm{mL})$ & $1208(591-1786)$ & $1276(714-1990)$ & $994(478-1406)$ & 0.09 \\
\hline Lactate dehydrogenase $(n=135)$ 120-240 (IU/L) & $455(328-572)$ & $458(343-633)$ & $398(276-515)$ & 0.05 \\
\hline IL-6 $(n=52)<17.4 \mathrm{pg} / \mathrm{mL}$ & $69.4(27.5-201.3)$ & $69.4(32.6-186.7)$ & $62.7(19.9-361)$ & 0.77 \\
\hline D-dimer $(n=142)<0.59 \mathrm{mg} / \mathrm{L}$ & $3.1(1.5-6.8)$ & $3.67(1.84-7.75)$ & $1.65(1.27-4.41)$ & 0.002 \\
\hline Disposition, n (\%) & & & & 0.03 \\
\hline Home (unassisted) & $62(42)$ & $40(37)$ & $22(55)$ & \\
\hline Skilled care facility & $47(32)$ & $41(38)$ & $6(15)$ & \\
\hline Death & $39(26)$ & $27(25)$ & $12(30)$ & \\
\hline
\end{tabular}

Institutional reference ranges are reported for laboratory values.

ICU, intensive care unit; IL-6, interleukin-6; WBC, white blood cell count.

or other indirect mechanisms, such as polypharmacy, systemic inflammatory responses and cerebrovascular events. Indeed, benzodiazepine sedation was common in this patient cohort, with nearly $60 \%$ of patients receiving midazolam at one point during ICU admission. Lorazepam was a common sedation agent as well, and benzodiazepine use is associated with delirium in critically ill patients. ${ }^{33-35}$ Whether benzodiazepine administration served as a driver of delirium or reflected worsening agitation (prompting additional sedative agents) remains unclear. Inflammation may have also contributed to delirium risk. Inflammatory markers (eg, c-reactive protein, ferritin, interleukin-6, lactate dehydrogenase) were considerably elevated in this patient cohort. In

Table 4 Postdischarge neuropsychological outcomes ${ }^{\star}$

\begin{tabular}{|c|c|c|c|}
\hline & All patients $(n=148)$ & Delirium ( $n=108)$ & No delirium $(n=40)$ \\
\hline Positive FAM-CAM, n (\%) $(n=17)$ & $4(24)$ & $4(31)$ & $0(0)$ \\
\hline PROMIS 4A cognitive abilities score, median (IQR) $(n=25)$ & $16(10-20)$ & $17(9-20)$ & $14(6)$ \\
\hline $\begin{array}{l}\text { Short blessed test-questionable cognitive impairment, } n(\%) \\
(n=22)\end{array}$ & $2(8.7)$ & $2(13)$ & $0(0)$ \\
\hline
\end{tabular}

Initial phone calls were placed between 30 and 60 days after hospital discharge, and the average time to survey completion was 83 days postdischarge.

${ }^{*}$ For each postdischarge survey, proportions are calculated based on the total numbers of surveys completed. In total, 25 surveys were completed for the PROMIS 4A test, 25 surveys were completed for the PHQ-9, 17 surveys were completed for the FAM-CAM and 22 surveys were completed for the Short Blessed Test. Reasons for not completing a test included the following: unable to contact ( $n=54)$, patient deceased $(n=43)$, refusal $(n=18)$, unable to provide consent $(n=5)$ and admission to skilled care facility $(n=3)$.

FAM-CAM, Family-based Confusion Assessment Method for delirium; PHQ, Patient Health Questionnaire; PROMIS, Patient-Reported Outcomes Measurement Information System. 


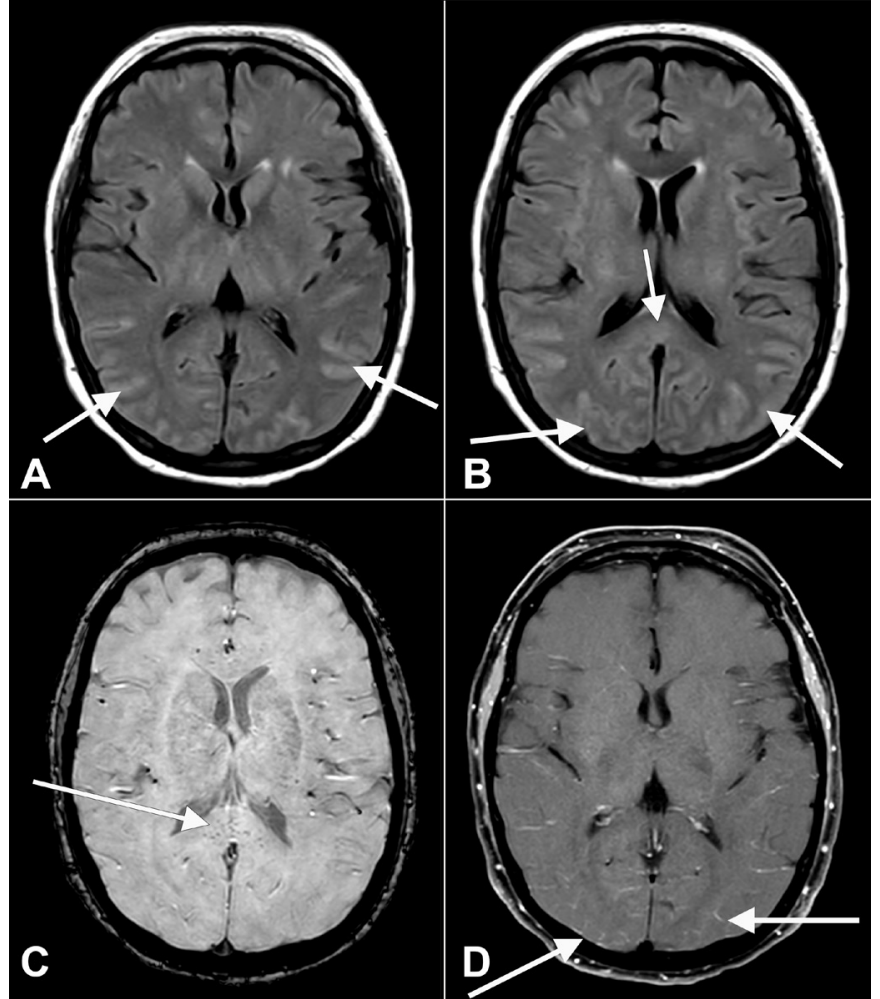

Figure 1 Axial fluid-attenuated inversion recovery (FLAIR) (A, B) images at the level of the basal ganglia show abnormal FLAIR hyperintense signal (arrows) affecting the bilateral occipital, temporal lobes. This appears almost sulcal suggesting a higher protein component within the cerebrospinal fluid. Note the elevated FLAIR signal in the splenium of the corpus callosum (arrow) suggesting parenchymal insult. Axial susceptibility weighted imaging (SWI) (C) at the level of the splenium of the corpus callosum shows small areas of susceptibility (arrow) in the splenium, likely related to microhaemorrhage. Axial T1 (D) postcontrast with fat suppression at the level of the basal ganglia shows subtle, though true, enhancement (arrows) in the posterior sulci, arachnoid pial (leptomeningeal) pattern suggesting a degree of encephalitis.

fact, serum levels observed in this study aligned withor exceeded-previously reported values in patients with severe COVID-19, ${ }^{25}$ and there was MRI evidence of neuroinflammation for at least two patients in this cohort. C-reactive protein was elevated in delirious patients, and c-reactive protein increases blood-brain barrier permeability in basic science models. ${ }^{36}$ However, this was an unadjusted, bivariable analysis and confounding remains possible. Cerebral ischaemia may also contribute to delirium in patients with COVID-19. Severe hypoxic-ischaemic injury occurred in a patient who experienced multiple cardiopulmonary arrests during the course of illness. Stroke has previously been reported in patients with COVID-19, ${ }^{37}$ as thromboembolic phenomena and cerebral malperfusion may both occur during the clinical course of COVID-19. Finally, overall illness severity may increase delirium risk. Indeed, patients with delirium had prolonged hospital and ICU lengths of stay, longer duration of mechanical ventilation and were more likely to require haemodialysis. Overall, multiple processes likely contribute to delirium in patients with COVID-19. Targeted case-control studies with appropriate statistical modelling strategies can be conducted to determine independent risk factors for delirium in this patient population.

Delirium prevention and management are inherently challenging for COVID-19 patients. While delirium prevention bundles have been demonstrated to reduce risk, ${ }^{38}{ }^{39}$ unique challenges posed by COVID-19 hinder the implementation of standard prevention practices. Spontaneous awakening and breathing trials, for example, may not have been possible due to illness severity and associated ventilator requirements. Clinicians may have also been limited in terms of sedation regimen. Agitation was commonly observed, and nearly $30 \%$ of patients required antipsychotics in this cohort. Agitation and hyperactive delirium likely prompted additional sedation and prolonged use of physical restraints. Early mobility was limited given illness severity, and family engagement was not possible due to visitor policy restrictions. In-person interactions with clinicians were also limited given the intent of reducing virus transmission. As such, the culmination of disease severity, limited face-to-face time spent with patients and visitor restriction policies likely hindered ICU liberation bundle implementation. Novel strategies for implementing delirium prevention bundles in this patient population may help to further mitigate risk and should be tested in prospective trials.

Neuropsychological impairment after discharge was also present for some patients based on subjective reporting, caretaker assessment and objective testing for depression and cognitive impairment. Furthermore, all patients who screened positive for possible impairment also experienced delirium in the hospital. These estimates may have been even higher, given that many patients called for postdischarge assessments were unable to be reached, refused participant or were still admitted to skilled care facilities. In fact, a large-scale, retrospective cohort study recently demonstrated an association between COVID-19 and subsequent neurologic and psychiatric impairment in the following 6 months, particularly for patients with severe illness. ${ }^{40}$ Whether postdischarge cognitive impairment is related specifically to COVID-19 or critical illness more broadly is unclear. Indeed, cognitive impairment is common at discharge for patients who experienced delirium while in the ICU, and delirium is present for nearly $20 \%$ of patients newly admitted to acute care facilities. $^{841}$ Moreover, cognitive impairment can be present for months-to-years after acute respiratory distress syndrome and sepsis, ${ }^{42-44}$ and symptoms of depression and post-traumatic stress disorder are commonly reported among ICU survivors. ${ }^{45}$ Neuropsychological impairment after discharge may thus, in part, reflect critical illness, rather than pathophysiologic insults specific to COVID-19. Additional research is needed to disambiguate these possibilities. Nonetheless, ICU patients with 
COVID-19 experience considerable neuropsychological burden both during and after hospitalisation. Ongoing longitudinal COVID-19 registry studies ${ }^{37}{ }^{46}$ will thus be important for (1) identifying such vulnerable patients who may require long-term care and resources and (2) understanding the underlying aetiology of cognitive decline in this patient population

The strengths of this study include identification of potential delirium risk factors, characterisation of delirium prevention strategies and postdischarge outcomes. Data were also representative of an academic tertiary care centre with nearly 150 patients. A validated, standardised chart review method was used to identify delirium ${ }^{23}$ and the study measures used to characterise cognitive function, such as the FAM-CAM, Short Blessed Test and PROMIS assessments, are standard measures that increase confidence in the results.

In terms of limitations, this was a single-centre analysis, and the results are restricted to the institution studied and ICUs only. The study was not conducted with a matched control cohort, as this was a descriptive analysis. Results from this study can be used to inform future study designs focused on identifying risk factors for delirium in this population. Delirium was assessed retrospectively for this study, and such retrospective techniques are not equivalent to prospective evaluation by an expert using gold-standard, Diagnostic and Statistical Manual of Mental Disorders criteria. ${ }^{47}$ Evidence for delirium reversal was also predicated on chart review and may have been underestimated. Delirium was also not differentiated from other encephalopathic states, which is neurobiologically challenging and outside of the scope of the current study. One patient was heavily sedated prior to death, and no formal delirium analysis was conducted. The postdischarge telephone-based assessments served as screening tools rather than thorough neuropsychological testing batteries. As such, these postdischarge results are preliminary and warrant rigorous, follow-up analysis. Additionally, neuropsychological impairment may have been present at baseline for some patients, particularly for those with previous neurological disorders. Baseline neuropsychological testing was not possible for this study. Finally, data were limited for postdischarge cognitive outcomes, as more than half of patients called were unavailable to complete assessments.

In summary, delirium is a common complication of COVID-19 with multiple contributing factors. Furthermore, neuropsychological impairment may persist in some patients after discharge. Further research should aim to identify independent risk factors in this population and novel, effective prevention strategies.

Acknowledgements We would like to acknowledge Dr Michael Kenes (PharmD, BCPS, BCCCP) and Ms Margaret Diehl for assistance with medical chart data extraction.

Contributors The study was originally conceived by JR, AM, MZ and PEV. Data acquisition was conducted by JR, AM, MZ, JB, MH-C, YA and PEV. Data analysis was conducted by JR, AM, MZ, JB, MH-C, MI, YA, GM and PEV. Final statistical analysis was conducted by GM and PEV. Neuroimaging studies were acquired and analysed by $\mathrm{RL}$, and the final neuroimaging figures were generated by RL and PEV. All authors contributed to the manuscript writing, critically reviewed the manuscript for intellectual content and approved the final manuscript.

Funding The authors have not declared a specific grant for this research from any funding agency in the public, commercial or not-for-profit sectors.

Competing interests None declared.

Patient consent for publication Not required.

Ethics approval Approval for this study was obtained from the University of Michigan Medical School Institutional Review Board (HUM00182646). A Health Insurance Portability and Accountability Act waiver was granted to retrospectively review patient medical records, and informed consent was not required for retrospective chart review. Patients who agreed to complete telephone surveys after discharge were consented over the telephone prior to survey administration using a comprehensive consent document. A waiver of documentation of consent was approved in conjunction with Institutional Review Board approval and as required by US Department of Health and Human Services regulations and policy.

Provenance and peer review Not commissioned; externally peer reviewed.

Data availability statement Data will be made available on request via Data Use Agreement. No additional unpublished data are available.

Supplemental material This content has been supplied by the author(s). It has not been vetted by BMJ Publishing Group Limited (BMJ) and may not have been peer-reviewed. Any opinions or recommendations discussed are solely those of the author(s) and are not endorsed by BMJ. BMJ disclaims all liability and responsibility arising from any reliance placed on the content. Where the content includes any translated material, BMJ does not warrant the accuracy and reliability of the translations (including but not limited to local regulations, clinical guidelines, terminology, drug names and drug dosages), and is not responsible for any error and/or omissions arising from translation and adaptation or otherwise.

Open access This is an open access article distributed in accordance with the Creative Commons Attribution Non Commercial (CC BY-NC 4.0) license, which permits others to distribute, remix, adapt, build upon this work non-commercially, and license their derivative works on different terms, provided the original work is properly cited, appropriate credit is given, any changes made indicated, and the use is non-commercial. See: http://creativecommons.org/licenses/by-nc/4.0/.

\section{ORCID iD}

Phillip E Vlisides http://orcid.org/0000-0003-3899-5536

\section{REFERENCES}

1 Helms J, Kremer S, Merdji H, et al. Neurologic features in severe SARS-CoV-2 infection. N Engl J Med 2020;382:2268-70.

2 Mao L, Jin H, Wang M, et al. Neurologic manifestations of hospitalized patients with coronavirus disease 2019 in Wuhan, China. JAMA Neurol 2020;77:683-90.

3 Varatharaj A, Thomas N, Ellul MA, et al. Neurological and neuropsychiatric complications of COVID-19 in 153 patients: a UKwide surveillance study. Lancet Psychiatry 2020;7:875-82.

4 Helms J, Kremer S, Merdji H, et al. Delirium and encephalopathy in severe COVID-19: a cohort analysis of ICU patients. Crit Care 2020;24:491.

5 Liotta EM, Batra A, Clark JR, et al. Frequent neurologic manifestations and encephalopathy-associated morbidity in COVID-19 patients. Ann Clin Trans/ Neurol 2020;7:2221-30.

6 Bergmann CC, Lane TE, Stohlman SA. Coronavirus infection of the central nervous system: host-virus stand-off. Nat Rev Microbiol 2006;4:121-32.

7 Pan Y, Yan J, Jiang Z, et al. Incidence, risk factors, and cumulative risk of delirium among ICU patients: a case-control study. Int J Nurs Sci 2019;6:247-51.

8 Ely EW, Shintani A, Truman B, et al. Delirium as a predictor of mortality in mechanically ventilated patients in the intensive care unit. JAMA 2004;291:1753-62.

9 Girard TD, Jackson JC, Pandharipande PP, et al. Delirium as a predictor of long-term cognitive impairment in survivors of critical illness. Crit Care Med 2010;38:1513-20.

10 Brummel NE, Jackson JC, Pandharipande PP, et al. Delirium in the ICU and subsequent long-term disability among survivors of mechanical ventilation. Crit Care Med 2014;42:369-77.

11 von Elm E, Altman DG, Egger M, et al. The strengthening the reporting of observational studies in epidemiology (STROBE) 
statement: guidelines for reporting observational studies. Lancet 2007;370:1453-7.

12 Siew ED, Fissell WH, Tripp CM, et al. Acute kidney injury as a risk factor for delirium and coma during critical illness. Am J Respir Crit Care Med 2017;195:1597-607.

13 Wintermann G-B, Rosendahl J, Weidner K, et al. Predictors of major depressive disorder following intensive care of chronically critically ill patients. Crit Care Res Pract 2018;2018:1586736.

14 Marra A, Ely EW, Pandharipande PP, et al. The ABCDEF bundle in critical care. Crit Care Clin 2017;33:225-43.

15 Pandharipande P, Banerjee A, McGrane S, et al. Liberation and animation for ventilated ICU patients: the ABCDE bundle for the back-end of critical care. Crit Care 2010;14:157.

16 Maybrier HR, Mickle AM, Escallier KE, et al. Reliability and accuracy of delirium assessments among investigators at multiple international centres. BMJ Open 2018;8:e023137.

17 Cella D, Yount S, Rothrock N, et al. The patient-reported outcomes measurement information system (PROMIS): progress of an $\mathrm{NIH}$ roadmap cooperative group during its first two years. Med Care 2007;45:S3-11.

18 Katzman R, Brown T, Fuld P, et al. Validation of a short orientationmemory-concentration test of cognitive impairment. Am J Psychiatry 1983;140:734-9.

19 Steis MR, Evans L, Hirschman KB, et al. Screening for delirium using family caregivers: Convergent validity of the family confusion assessment method and interviewer-rated confusion assessment method. J Am Geriatr Soc 2012;60:2121-6.

20 Kroenke K, Spitzer RL, Williams JB. The PHQ-9: validity of a brief depression severity measure. J Gen Intern Med 2001;16:606-13.

21 Levis B, Benedetti A, Thombs BD, et al. Accuracy of patient health questionnaire-9 (PHQ-9) for screening to detect major depression: individual participant data meta-analysis. BMJ 2019;365:11476.

22 Inouye SK, van Dyck CH, Alessi CA, et al. Clarifying confusion: the confusion assessment method. A new method for detection of delirium. Ann Intern Med 1990;113:941-8.

23 Inouye SK, Leo-Summers L, Zhang Y, et al. A chart-based method for identification of delirium: validation compared with interviewer ratings using the confusion assessment method. J Am Geriatr Soc 2005;53:312-8.

24 Hsieh SJ, Soto GJ, Hope AA, et al. The association between acute respiratory distress syndrome, delirium, and in-hospital mortality in intensive care unit patients. Am J Respir Crit Care Med 2015;191:71-8.

25 Brück E, Schandl A, Bottai M, et al. The impact of sepsis, delirium, and psychological distress on self-rated cognitive function in ICU survivors-a prospective cohort study. J Intensive Care 2018;6:2.

26 Gamberini M, Bolliger D, Lurati Buse GA, et al. Rivastigmine for the prevention of postoperative delirium in elderly patients undergoing elective cardiac surgery-a randomized controlled trial. Crit Care Med 2009;37:1762-8

27 Ely EW, Girard TD, Shintani AK, et al. Apolipoprotein E4 polymorphism as a genetic predisposition to delirium in critically ill patients. Crit Care Med 2007;35:112-7.

28 Price-Haywood EG, Burton J, Fort D, et al. Hospitalization and mortality among black patients and white patients with COVID-19. N Engl J Med 2020;382:2534-43.
29 Millett GA, Jones AT, Benkeser D, et al. Assessing differential impacts of COVID-19 on black communities. Ann Epidemiol 2020;47:37-44.

30 Huizinga GP, Singer BH, Singer K. The collision of meta-inflammation and SARS-CoV-2 pandemic infection. Endocrinology 2020;161 doi:10.1210/endocr/bqaa154

31 Oh ES, Sieber FE, Leoutsakos J-M, et al. Sex differences in hip fracture surgery: preoperative risk factors for delirium and postoperative outcomes. J Am Geriatr Soc 2016;64:1616-21.

32 Elie M, Cole MG, Primeau FJ, et al. Delirium risk factors in elderly hospitalized patients. J Gen Intern Med 1998;13:204-12.

33 Pisani MA, Murphy TE, Araujo KLB, et al. Benzodiazepine and opioid use and the duration of intensive care unit delirium in an older population. Crit Care Med 2009;37:177-83.

34 Zaal IJ, Devlin JW, Hazelbag M, et al. Benzodiazepine-associated delirium in critically ill adults. Intensive Care Med 2015;41:2130-7.

35 Pandharipande P, Shintani A, Peterson J, et al. Lorazepam is an independent risk factor for transitioning to delirium in intensive care unit patients. Anesthesiology 2006;104:21-6.

36 Hsuchou H, Kastin AJ, Mishra PK, et al. C-reactive protein increases BBB permeability: implications for obesity and neuroinflammation. Cell Physiol Biochem 2012;30:1109-19.

37 Ntaios G, Michel P, Georgiopoulos G, et al. Characteristics and outcomes in patients with COVID-19 and acute ischemic stroke: the global COVID-19 stroke registry. Stroke 2020;51:e254-8.

38 Ely EW. The ABCDEF bundle: science and philosophy of how ICU liberation serves patients and families. Crit Care Med 2017;45:321-30.

39 Barnes-Daly MA, Phillips G, Ely EW. Improving hospital survival and reducing brain dysfunction at seven California community hospitals: implementing PAD guidelines via the ABCDEF bundle in 6,064 patients. Crit Care Med 2017;45:171-8.

40 Taquet M, Geddes JR, Husain M, et al. 6-month neurological and psychiatric outcomes in 236379 survivors of COVID-19: a retrospective cohort study using electronic health records. Lancet Psychiatry 2021;8:416-27.

41 Kiely DK, Bergmann MA, Murphy KM, et al. Delirium among newly admitted postacute facility patients: prevalence, symptoms, and severity. J Gerontol A Biol Sci Med Sci 2003;58:M441-5.

42 Hopkins RO, Weaver LK, Collingridge D, et al. Two-year cognitive, emotional, and quality-of-life outcomes in acute respiratory distress syndrome. Am J Respir Crit Care Med 2005;171:340-7.

43 Ehlenbach WJ, Hough CL, Crane PK, et al. Association between acute care and critical illness hospitalization and cognitive function in older adults. JAMA 2010;303:763-70.

44 Iwashyna TJ, Ely EW, Smith DM, et al. Long-term cognitive impairment and functional disability among survivors of severe sepsis. JAMA 2010;304:1787-94.

45 Hatch R, Young D, Barber V, et al. Anxiety, depression and post traumatic stress disorder after critical illness: a UK-wide prospective cohort study. Crit Care 2018;22:310.

46 Román GC, Reis J, Spencer PS, et al. COVID-19 international neurological registries. Lancet Neurol 2020;19:484-5.

47 American Psychiatric Association. Diagnostic and statistical manual of mental disorders. 5th edn. Arlington, VA, 2013. 